\title{
Design of Robust Fuzzy Sliding-Mode Controller for a Class of Uncertain Takagi-Sugeno Nonlinear Systems
}

\author{
X.Z. Zhang, Y.N. Wang
}

\author{
X.Z. Zhang* \\ 1. School of Electrical and Information Engineering \\ Hunan University \\ China, 410082 Changsha, Yuelushan \\ 2. Cooperative Innovative Center of Wind Power \\ Hunan Institute of Engineering \\ China, 411104 Xiangtan, Fuxing Road 88 \\ *Corresponding author: zxz@hnie.edu.cn \\ Y.N. Wang \\ School of Electrical and Information Engineering \\ Hunan University \\ China, 410082 Changsha, Yuelushan \\ yaonan@hnu.edu.cn
}

\begin{abstract}
This paper presents the fuzzy design of sliding mode control (SMC) for nonlinear systems with uncertainties, which can be represented by a Takagi-Sugeno (T-S) model. There exist the parameter uncertainties in both state and input matrices, as well as the matched external disturbance. The key feature of this work is the great ability of the controller to deal with systems without assuming that the control matrices of each local T-S model to be same and knowing the priori information of the upper norm-bounds of uncertainties. A sufficient condition for the existence of the desired fuzzy SMC is obtained by solving a set of linear matrix inequalities (LMIs). The reachability of the specified sliding surface is proven. A numerical example is illustrated in order to show the validity of the proposed scheme.

Keywords: uncertain T-S nonlinear system, sliding mode control, linear matrix inequalities (LMI), system stabilitybooks, examples.
\end{abstract}

\section{Introduction}

It has been shown that Takagi Sugeno (T-S) fuzzy model is a powerful and efficient tool to handle complex nonlinear system, and be employed in most model-based fuzzy analysis approaches $[1,2]$. Since the Parallel Distributed compensation (PDC) approach is formulated by Tanaka and Wang [3], the stability analysis and stabilized controller design for T-S fuzzy systems become achievable and have been applied in a wide range of areas such as electrical/mechanical systems [4,5], process control [6], robot [7] and time-delayed systems [8]. A lot of research outcomes on this field have been appeared in literatures. Practically, a real nonlinear system may contain various kinds of uncertainties such as parameter variations, modeling errors and external disturbances, etc. In this case, the PDC-based controllers [3] may perform well no longer. Then, the robustly stabilized control to uncertainties of nonlinear fuzzy systems is required. Based on the T-S fuzzy model, $\mathrm{Wu}$ studied the robust $H_{2}$ fuzzy observer-based control problem for discrete-time nonlinear systems with parametric uncertainties [9]. Lin, et.al, investigated the mixed $H_{2} / H_{\infty}$ controller for uncertain T-S fuzzy systems with discrete time-varying delay [10].

It's well known that the sliding mode control (SMC) is an effective means to design robust controllers for nonlinear systems with uncertainties bounded by known scalar-valued functions. Recently, the SMC has been successfully applied into solving the stabilization problems for uncertain fuzzy systems $[11,12]$. Specially, under the SMC control the state trajectories would 
enter into the pre-designed sliding-mode motion within finite time and after that keep staying on it; thus, the system dynamics is not sensitive to parameter variations and external disturbances any more $[9,13]$. Basically, the feedback gains of the SMC control are often determined by the feasible solution of a set of Linear Matrix Inequalities (LMI). Choi presented a robust stabilization of uncertain fuzzy systems using SMC system approach [14]. Zhang and Wang proposed a mixed SMC- $H_{\infty}$ controller for time-delay system with unmatched uncertainties [15].

Although many researchers have proposed a variety of T-S fuzzy SMC-based methods $[4,7-$ 10,14 ], by far there still two problems yet remained to be well-solved: (1) the suitable relaxation on the assumption that all the control matrices of the nominal sub-systems' models are identical; It is practically difficult to satisfy this assumption. In practice, this assumption is very strict and insufficient to model various uncertainties/nonlinearities in most of actual systems such as nonlinear stirred tank reactor, fourth-order cart-pole system, and active queue management in TCP networks and two-link robot manipulator; (2) the reasonable assumption that the system uncertainties/perturbations are unknown but norm-bounded. For the SMC-based methods, if uncertainties are known, it's easy to choose proper switching gains, which are bigger than the upper norm-bounds of uncertainties, to ensure the reachability of sliding-mode motion. Unfortunately, the information of the upper bound of uncertainties/perturbations may not easily be obtained in practice. Therefore, it's supposed to adopt parameter identification or adaptive control approach to estimate the bounds of uncertainties on-line [16].

Motivated by the above discussion, it is meaningful to design fuzzy variable structure controller in this paper such that the closed-loop system is asymptotically stable without (1) assuming the control matrices of each local linear model to be same; and (2) knowing the priori information of the upper norm-bounds of uncertainties. The existence condition of linear sliding surfaces and the asymptotical stability of the reduced-order equivalent sliding-mode dynamics are firstly derived by the LMI optimization technique. After the establishment of the local T-S model, the upper norm-bounds of uncertainties and modeling errors between the original and local systems are estimated. Then, the designed SMC controller composes of a state-feedback control term and an adaptive switching-feedback control term, which are achieved based on the Lyapunov function method. As a result, the aforementioned problems are well solved in the proposed scheme. Finally, a numerical example is illustrated in order to show the effectiveness of the proposed methods.

\section{Problem formulation and preliminaries}

As stated in Introduction, T-S fuzzy models can provide an effective representation of complex nonlinear systems in terms of fuzzy sets and fuzzy reasoning applied to a set of linear input output sub-models. Hence, in this work, a class of nonlinear systems is represented by a T-S model. As in [3], the T-S fuzzy system with uncertainties is described by fuzzy IF THEN rules, which locally represent linear input-output relations of nonlinear systems.

The $i$-th rule of the fuzzy model is formulated in the following equation:

Plant rule $i$ : IF $z_{1}$ is $M_{1}^{i}$ and $z_{2}$ is $M_{2}^{i} \cdots$ and $z_{p}$ is $M_{1}^{p}$, THEN

$$
\dot{\boldsymbol{x}}(t)=\boldsymbol{A}_{i} \boldsymbol{x}(t)+\boldsymbol{B}_{i} \boldsymbol{u}(t)
$$

where $M_{j}^{i}$ is the fuzzy set, $\boldsymbol{z}(t)=\left[z_{1}(t), z_{2}(t), \cdots, z_{p}(t)\right]^{T}$ is the premise variable vector, $r$ is the number of rules of this T-S fuzzy model. $\boldsymbol{x}(t) \in \mathbb{R}^{n}$ is the state vector, $\boldsymbol{u}(t) \in \mathbb{R}^{m}$ is the control input vector. $\boldsymbol{A}_{i}, \boldsymbol{B}_{i}$ are known real constant matrices with appropriate dimensions. 
The overall fuzzy model achieved by fuzzy blending of each plant rule is represented as follows:

$$
\dot{\boldsymbol{x}}(t)=\sum_{i=1}^{r} h_{i}(\boldsymbol{z})\left[\boldsymbol{A}_{i} \boldsymbol{x}(t)+\boldsymbol{B}_{i} \boldsymbol{u}(t)\right]
$$

where $h_{i}(\boldsymbol{z}(t))=\frac{w_{i}(\boldsymbol{z}(t))}{\sum_{j=1}^{r} w_{i}(\boldsymbol{z}(t))}, w_{i}(\boldsymbol{z}(t))=\prod_{j=1}^{p} M_{j}^{i}(\boldsymbol{z}(t))$, in which $M_{j}^{i}(\boldsymbol{z}(t))$ is the membership grade of $z_{j}(t)$ in $M_{j}^{i}$. According to the theory of fuzzy sets, we have $M_{j}^{i}(\boldsymbol{z}(t)) \geq 0$. Therefore, it implies that $h_{i}(\boldsymbol{z}(t)) \geq 0$ and $\sum_{i=1}^{r} h_{i}(\boldsymbol{z}(t))=1$.

In practical applications, the system (2) usually exists the parameters variation and the external disturbances. After considering the perturbation, the global fuzzy model of system (2) can be rewritten as

$$
\dot{\boldsymbol{x}}(t)=\sum_{i=1}^{r} h_{i}(\boldsymbol{z})\left[\left(\boldsymbol{A}_{i}+\Delta \boldsymbol{A}_{i}\right) \boldsymbol{x}(t)+\left(\boldsymbol{B}_{i}+\Delta \boldsymbol{B}_{i}\right) \boldsymbol{u}(t)+d_{i}(t, \boldsymbol{x}, \boldsymbol{u})\right]
$$

where $\Delta \boldsymbol{A}_{i}, \Delta \boldsymbol{B}_{i}$ are unknown time-varying matrices representing parameter uncertainties, and $d_{i}(t, \boldsymbol{x}, \boldsymbol{u})$ denotes the external disturbance.

In this work, the control matrices doesn't satisfy $\boldsymbol{B}_{1}=\boldsymbol{B}_{2}=\ldots=\boldsymbol{B}_{r}$ and we define the weighted nominal matrix as $\boldsymbol{B}=\sum_{i=1}^{r} h_{i}(\boldsymbol{z}) \boldsymbol{B}_{i}$. Moreover, it is required that $\boldsymbol{B}$ is a nonzero matrix with full-column (or full-row) rank. The uncertain matrices are assumed to be matched, i.e. there exist certain functions $\boldsymbol{D}(t), \boldsymbol{E}(t)$ and $\boldsymbol{F}(t)$ such that $\Delta \boldsymbol{A}_{i}(t)=\boldsymbol{B}_{i} \boldsymbol{D}(t)$, $\Delta \boldsymbol{B}_{i}(t)=\boldsymbol{B}_{i} \boldsymbol{E}(t)$ and $d_{i}(t, \boldsymbol{x}, \boldsymbol{u})=\boldsymbol{B}_{i} \boldsymbol{F}(t)$ hold. As a result, the system (3) can be rewritten as

$$
\dot{\boldsymbol{x}}(t)=\sum_{i=1}^{r} h_{i}(\boldsymbol{z}) \boldsymbol{A}_{i} \boldsymbol{x}(t)+\boldsymbol{B}[\boldsymbol{u}(t)+g(t, \boldsymbol{x}, \boldsymbol{u})]
$$

where the time-varying function $g(t, \boldsymbol{x}, \boldsymbol{u})=\boldsymbol{D}(t) \boldsymbol{x}(t)+\boldsymbol{E}(t) \boldsymbol{u}(t)+\boldsymbol{F}(t)$, then $g(t, \boldsymbol{x}, \boldsymbol{u})$ contains all the perturbation in system (3). Before proceeding, some assumptions and lemmas are given as following, which are useful for the development of our result.

(Assumption.1) The time-varying uncertainties $g(t, \boldsymbol{x}, \boldsymbol{u})$ is assumed to be norm-bounded, that is, $\|g\| \leq \sum_{k=0}^{N-1} \gamma_{k}\|\boldsymbol{x}(t)\|^{k}$, where $\gamma_{k}$ is unknown coefficient and $N$ is a positive integer.

Remark 1: Assumption 1 is a standard assumption in the study of variable structure control.

(Lemma.1 Choi [14]): Give any matrix $X, Y, Z$ with appropriate dimensions, and $Y>0$. Then we have $-X^{T} Z-Z^{T} X \leq X^{T} Y X+Z^{T} Y^{-1} Z$.

(Lemma.2 Schur's Complement [3]): Given the matrix inequality $\left[\begin{array}{cc}S_{11} & S_{12} \\ S_{12}^{T} & S_{22}\end{array}\right]<0$, where $S_{11}$ and $S_{22}$ are invertible symmetrical matrices, it's equal to each of the following inequalities:

(i) $S_{11}<0, S_{22}-S_{12}^{T} S_{11}^{-1} S_{12}<0$; (ii) $S_{22}<0, S_{11}-S_{12} S_{22}^{-1} S_{12}^{T}<0$.

\section{Controller design}

The objective of this work is to design an SMC law such that the desired control performance for the resulting closed-loop system is obtained despite of parameter uncertainties and unmatched external disturbance. In this section, an SMC law is synthesized such that the closed-loop systems are robustly asymptotically stable. It is also proven that the reachability of the specified switching (sliding) surface $S(t)=0$ can be ensured by the proposed SMC law. Thus, it is concluded that the synthesized SMC law can guarantee the state trajectories of uncertain system (4) to be driven onto the sliding surface, and asymptotically tend to zero along the specified sliding surface. 


\subsection{Design of the sliding function and stability analysis of the sliding motion}

Essentially, a VSC design is composed of two phases: hyper-plane design and controller design [9]. There are various methods for designing hyper-plane, however in this paper the switching surface is defined as

$$
S(t, \boldsymbol{x})=\boldsymbol{C} \boldsymbol{x}(t)
$$

where $\boldsymbol{C} \in \mathbb{R}^{m \times n}$ is the designed coefficient. According to the previous works [2], for the system (2), there are two prerequisites to find the switching surface (5) that is

(P-1): The matrix $\boldsymbol{C} \boldsymbol{B}$ is invertible for any $h_{i}(\boldsymbol{z})$ satisfying $h_{i}(\boldsymbol{z}) \geq 0$ and $\sum_{i=1}^{r} h_{i}(\boldsymbol{z})=1$.

(P-2): The reduced sliding-mode motion of the system dynamics restricted on the switching surface is asymptotically stable to all admissible uncertainties.

In this part, we analyze the dynamic performance of the system described by (4), and derives some sufficient conditions for the asymptotically stability of the sliding dynamics via LMI method. The following theorem shows that system (4) with the switching surface as in (5) is asymptotically stable.

Theorem 1. Consider the fuzzy uncertain systems (4) with Assumptions (1). The switching function is given by (5). If there is feasible solution $Q$ such that the LMIs shown in (6) hold for $\forall i, j, k \in 1, \ldots, r$, the proper sliding-mode coefficient $C$ exists and $C=\left(B^{T} Q^{-1} B\right)^{-1} B^{T}$.

$$
\left[\begin{array}{ccc}
\boldsymbol{A}_{i} \boldsymbol{Q}+\boldsymbol{Q} \boldsymbol{A}_{i}^{T} & \boldsymbol{Q} \boldsymbol{B}_{j}^{T} & \boldsymbol{A}_{i}^{T} \\
\boldsymbol{B}_{j}^{T} \boldsymbol{Q} & -\boldsymbol{B}_{j}^{T} \boldsymbol{B}_{k} & 0 \\
\boldsymbol{A}_{i} & 0 & -\boldsymbol{I}
\end{array}\right]<0
$$

where the invertible matrix $Q \in \mathbb{R}^{n \times n}$ is decisive variable.

Proof: First, the existence of the coefficient $C$ is proved. Since $Q$ is designed to be invertible, the inequalities $\boldsymbol{B}^{T} \boldsymbol{B} \neq 0$ and $\boldsymbol{B}^{T} \boldsymbol{Q}^{-1} \boldsymbol{B} \neq 0$ hold; thus, its easy to prove that the achieved matrix $\boldsymbol{C} \boldsymbol{B}$ is invertible and $(\boldsymbol{C} \boldsymbol{B})^{-1}=\left(\boldsymbol{B}^{T} \boldsymbol{B}\right)^{-1}\left(\boldsymbol{B}^{T} \boldsymbol{Q}^{-1} \boldsymbol{B}\right)$. That is to say, if the coefficient is chosen as $\boldsymbol{C}=\left(\boldsymbol{B}^{T} \boldsymbol{Q}^{-1} \boldsymbol{B}\right) \boldsymbol{B}^{T}$, both the sliding surface and the equivalent control exist. With the chosen sliding surface, once the $n$-order system enters the $m$-order sliding surface $S=\boldsymbol{C} \boldsymbol{x}(t)$, the system dynamic of (4) is equivalent to the $(n-m)$-order sliding motion, and the system states will asymptotically converge to zero with proper switching gains. In the following, we will derive the equivalent control and the sliding mode as well as the stability analysis.

Now, the linear transformation $\boldsymbol{T}$ is carried on the states to separate the $m$-th sliding-mode states and the reduced $(n-m)$-order states

$$
\boldsymbol{T}=\left[\begin{array}{c}
T_{1} \\
T_{2}
\end{array}\right]=\left[\begin{array}{c}
\left(\boldsymbol{K}^{T} \boldsymbol{Q} \boldsymbol{K}\right)^{-1} \boldsymbol{K}^{T} \boldsymbol{Q} \\
\left(\boldsymbol{B}^{T} \boldsymbol{Q}^{-1} \boldsymbol{B}\right)^{-1} \boldsymbol{B}^{T}
\end{array}\right]
$$

where $T_{1} \in \mathbb{R}^{(n-m) \times n}, T_{2} \in \mathbb{R}^{m \times n}$ and $\boldsymbol{K} \in \mathbb{R}^{n \times(n-m}$ is an orthonormal basis for the null space of $\boldsymbol{B}^{T}$ such that $\boldsymbol{B}^{T} \boldsymbol{K}=0$ and $\boldsymbol{K}^{T} \boldsymbol{K}=\boldsymbol{I}$ satisfy. We have

$$
\boldsymbol{z}=\boldsymbol{T} \boldsymbol{x}(t)=\left[\begin{array}{l}
z_{1} \\
z_{2}
\end{array}\right]
$$

In (8), it's obvious that $z_{2}=\left(\boldsymbol{B}^{T} \boldsymbol{Q}^{-1} \boldsymbol{B}\right) \boldsymbol{B}^{T} \boldsymbol{x}(t)=\boldsymbol{C} \boldsymbol{x}(t)$ is the sliding-mode state and $z_{1}$ is the reduced-order state. Its easy to obtain the inverse transformation $\boldsymbol{T}^{-1}=\left[\boldsymbol{K}, \boldsymbol{Q}^{-1} \boldsymbol{B}\right]$. 
By differentiating the above transformation, we can obtain

$$
\begin{aligned}
\dot{z}= & \boldsymbol{T} \boldsymbol{x}(t)=\sum_{i=1}^{r} h_{i}(\boldsymbol{z})\left[\begin{array}{c}
\boldsymbol{T}_{1} \\
\boldsymbol{T}_{2}
\end{array}\right] \boldsymbol{A}_{i}\left[\begin{array}{ll}
\boldsymbol{K} & \boldsymbol{Q}^{-1} \boldsymbol{B}
\end{array}\right] \boldsymbol{T} \boldsymbol{x}(t)+\left[\begin{array}{l}
\boldsymbol{T}_{1} \\
\boldsymbol{T}_{2}
\end{array}\right] \boldsymbol{B}[\boldsymbol{u}+g(t, \boldsymbol{x}, \boldsymbol{u})] \\
= & \sum_{i=1}^{r} h_{i}(\boldsymbol{z})\left[\begin{array}{ll}
\boldsymbol{T}_{1} \boldsymbol{A}_{i} \boldsymbol{K} & \boldsymbol{T}_{1} \boldsymbol{A}_{i} \boldsymbol{Q}^{-1} \boldsymbol{B} \\
\boldsymbol{T}_{2} \boldsymbol{A}_{i} \boldsymbol{K} & \boldsymbol{T}_{2} \boldsymbol{A}_{i} \boldsymbol{Q}^{-1} \boldsymbol{B}
\end{array}\right]\left[\begin{array}{c}
z_{1} \\
z_{2}
\end{array}\right]+\left[\begin{array}{c}
\boldsymbol{T}_{1} \boldsymbol{B} \\
\boldsymbol{T}_{2} \boldsymbol{B}
\end{array}\right][\boldsymbol{u}+g(t, \boldsymbol{x}, \boldsymbol{u})]
\end{aligned}
$$

In (9), it's not difficult to verify that $\boldsymbol{T}_{1} \boldsymbol{B}=\left(\boldsymbol{K} \boldsymbol{Q}^{-1} \boldsymbol{K}\right) \boldsymbol{K}^{T} \boldsymbol{B}=0$ and $\boldsymbol{T}_{2} \boldsymbol{B}=\boldsymbol{C} \boldsymbol{B}$. According to the sliding mode theory, let $\dot{S}=S=\dot{z}_{2}=0$, we have

$$
\begin{aligned}
\dot{z}_{2}= & \sum_{i=1}^{r} h_{i}(\boldsymbol{z})\left(\boldsymbol{T}_{2} \boldsymbol{A}_{i} \boldsymbol{K} z_{1}+\boldsymbol{T}_{2} \boldsymbol{A}_{i} \boldsymbol{Q}^{-1} \boldsymbol{B} z_{2}\right)+\boldsymbol{T}_{2} \boldsymbol{B}[\boldsymbol{u}+g(t, \boldsymbol{x}, \boldsymbol{u})] \\
= & \sum_{i=1}^{r} h_{i} \boldsymbol{C} \boldsymbol{A}_{i} \boldsymbol{x}(t)+\boldsymbol{T}_{2} \boldsymbol{B}[g(t, \boldsymbol{x}, \boldsymbol{u})]
\end{aligned}
$$

From (16), the following equivalent control can be derived

$$
\boldsymbol{u}_{e q}(t)=-g(t, \boldsymbol{x}, \boldsymbol{u})-\sum_{i=1}^{r} h_{i}((z)(t))(\boldsymbol{C B})^{-1} \boldsymbol{C} \boldsymbol{A}_{i} \boldsymbol{x}(t)
$$

Substitute $\boldsymbol{u}_{e q}(t)$ in (11) into (10), the reduced-order sliding motion in the switching surface can be obtained as

$$
\dot{z}_{1}=\sum_{i=1}^{r} h_{i}(\boldsymbol{z}(t))\left(\boldsymbol{K}^{T} \boldsymbol{Q} \boldsymbol{K}\right)^{-1} \boldsymbol{K}^{T}\left[\boldsymbol{I}-\boldsymbol{B}\left(\boldsymbol{C} \boldsymbol{B}^{-1} \boldsymbol{C}\right)\right] \boldsymbol{A}_{i} \boldsymbol{K} z_{1}
$$

To analyze the stability of the sliding-mode dynamics (8), we consider the fuzzy uncertain system (4) with LMIs in (6) and choose the Lyapunov functional candidate $V_{1}=z_{1}^{T} \boldsymbol{P} z_{1}$, where $\boldsymbol{P}=\boldsymbol{K}^{T} \boldsymbol{Q} \boldsymbol{K}$ is a positive matrix.

By differentiating the function $\boldsymbol{V}_{1}$, we obtain the differential along the trajectories as

$$
\begin{aligned}
\dot{V}_{1}= & z_{1}^{T} \boldsymbol{P}_{1}+\dot{z}_{1}^{T} \boldsymbol{P} z_{1} \\
& =\sum_{i=1}^{r} h_{i}(z)\left[z_{1}^{T} \boldsymbol{P} \boldsymbol{T}_{1}\left(\boldsymbol{I}-\boldsymbol{B}(\boldsymbol{C B})^{-1} \boldsymbol{C} \boldsymbol{A}_{i}\right) \boldsymbol{K} z_{1}+z_{1}^{T} \boldsymbol{K}^{T} \boldsymbol{A}_{i}^{T}\left(\boldsymbol{I}-\boldsymbol{B}(\boldsymbol{C B})^{-1} \boldsymbol{C}\right)^{T} \boldsymbol{T}_{1}^{T} \boldsymbol{P}^{T} z_{1}\right] \\
& =\sum_{i=1}^{r} h_{i}(z)\left[z_{1}^{T} \boldsymbol{K}^{T} \boldsymbol{Q}\left(\boldsymbol{I}-\boldsymbol{B}(\boldsymbol{C B})^{-1} \boldsymbol{C}\right) \boldsymbol{A}_{i} \boldsymbol{K} z_{1}+(*)\right] \\
& =\sum_{i=1}^{r} h_{i}(z)\left[w^{T}(t) \boldsymbol{Q}\left(\boldsymbol{I}-\boldsymbol{B}(\boldsymbol{C B})^{-1} \boldsymbol{C}\right) \boldsymbol{A}_{i} \boldsymbol{K} w(t)+(*)\right] \\
& =\sum_{i=1}^{r} h_{i}(z) w^{T}(t)\left\{\left[\boldsymbol{Q} \boldsymbol{A}_{i}+(*)\right]-\left[\boldsymbol{Q B}(\boldsymbol{C B})^{-1} \boldsymbol{C} \boldsymbol{A}_{i}+(*)\right]\right\} w(t)
\end{aligned}
$$

where the defined new state vector $w(t)=\boldsymbol{K} z_{1} \in \mathbb{R}^{n \times 1}$.

By now, the stability of the states $\boldsymbol{x}(t)$ in the switching surface is equivalent to the stability of the news states $\boldsymbol{w}(t)$. Noticing that $\boldsymbol{B}(\boldsymbol{C} \boldsymbol{B})^{-1} \boldsymbol{C}=\boldsymbol{B}\left(\boldsymbol{B}^{T} \boldsymbol{B}\right)^{-1} \boldsymbol{B}^{T}$, it is easy to have $\dot{V}_{1}<0$ if the following inequality holds

$$
\left[\boldsymbol{Q} \boldsymbol{A}_{i}+(*)\right]-\boldsymbol{Q} \boldsymbol{B}\left(\boldsymbol{B}^{T} \boldsymbol{B}\right)^{-1} \boldsymbol{B}^{T} \boldsymbol{A}_{i}-\boldsymbol{A}_{i}^{T} \boldsymbol{B}\left(\boldsymbol{B}^{T} \boldsymbol{B}\right)^{-1} \boldsymbol{B}^{T} \boldsymbol{Q} \leq 0
$$


According to Lemma 2, let $\boldsymbol{X}=\boldsymbol{Q} \boldsymbol{B}\left(\boldsymbol{B}^{T} \boldsymbol{B}\right)^{-1} \boldsymbol{B}^{T}, \boldsymbol{Z}=\boldsymbol{A}_{i}$ and $\boldsymbol{Y}=\boldsymbol{I}>0$, the inequality in (21) implies that

$$
\left[\boldsymbol{Q} \boldsymbol{A}_{i}+(*)\right]+\boldsymbol{Q} \boldsymbol{B}\left(\boldsymbol{B}^{T} \boldsymbol{B}\right)^{-1} \boldsymbol{B}^{T}+\boldsymbol{A}_{i}^{T} \boldsymbol{A}_{i} \leq 0
$$

According to the inequalities (14), (15), we have

$$
\left[\boldsymbol{Q} \boldsymbol{A}_{i}+(*)\right]-\left[\boldsymbol{Q} \boldsymbol{B}\left(\boldsymbol{B}^{T} \boldsymbol{B}\right)^{-1} \boldsymbol{B}^{T} \boldsymbol{A}_{i}+\boldsymbol{A}_{i}^{T} \boldsymbol{B}^{T}\left(\boldsymbol{B}^{T} \boldsymbol{B}\right)^{-1} \boldsymbol{B}^{T} \boldsymbol{Q}\right] \leq 0
$$

By using Schur's complement formula and noticing that $\boldsymbol{B}^{T} \boldsymbol{B} \geq 0$, we can rewrite the above inequality as

$$
\left[\begin{array}{ccc}
\boldsymbol{A}_{i} \boldsymbol{Q}+\boldsymbol{Q} \boldsymbol{A}_{i}^{T} & \boldsymbol{Q} \boldsymbol{B} & \boldsymbol{A}_{i}^{T} \\
\boldsymbol{B}^{T} \boldsymbol{Q} & -\boldsymbol{B}^{T} \boldsymbol{B} & 0 \\
\boldsymbol{A}_{i} & 0 & -\boldsymbol{I}
\end{array}\right]<0
$$

From (17) and noticing the definition of the matrix $\boldsymbol{B}$ and $\boldsymbol{B}^{T} \boldsymbol{B}=\sum_{j=1}^{r} \sum_{k=1}^{r} h_{j}(z) h_{k}(z) \boldsymbol{B}_{j}^{T} \boldsymbol{B}_{k}$, we can deduce the following inequality

$$
\dot{V}_{1}=\sum_{i=1}^{r} \sum_{j=1}^{r} \sum_{k=1}^{r} h_{i}(z) h_{j}(z) h_{k}(z) w^{T}(t)\left[\begin{array}{ccc}
\boldsymbol{A}_{i} \boldsymbol{Q}+\boldsymbol{Q} \boldsymbol{A}_{i}^{T} & \boldsymbol{Q} \boldsymbol{B}_{j}^{T} & \boldsymbol{A}_{i}^{T} \\
\boldsymbol{B}_{j}^{T} \boldsymbol{Q} & -\boldsymbol{B}_{j}^{T} \boldsymbol{B}_{k} & 0 \\
\boldsymbol{A}_{i} & 0 & -\boldsymbol{I}
\end{array}\right] w(t) \leq 0
$$

and this leads to for $\forall i, j, k \in 1, \ldots, r$

$$
\left[\begin{array}{ccc}
\boldsymbol{A}_{i} \boldsymbol{Q}+\boldsymbol{Q} \boldsymbol{A}_{i}^{T} & \boldsymbol{Q B}_{j}^{T} & \boldsymbol{A}_{i}^{T} \\
\boldsymbol{B}_{j}^{T} \boldsymbol{Q} & -\boldsymbol{B}_{j}^{T} \boldsymbol{B}_{k} & 0 \\
\boldsymbol{A}_{i} & 0 & -\boldsymbol{I}
\end{array}\right] w(t) \leq 0
$$

This means that the sliding surface (5) and the equivalent control (11) for the fuzzy system (4) with Assumption 1 exist and the sliding motion is asymptotically stable.

\subsection{Design of controller and adaptive laws}

As the last step of design procedure, we will further design the VSC controller ensures the reachability of the specified switching surface. The adaptive VSC controller is represented in a set of fuzzy rules as following

Controller rule $i$ : IF $z_{1}$ is $M_{1}^{i}$ and $z_{2}$ is $M_{2}^{i} \cdots$ and $z_{p}$ is $M_{p}^{i}$, THEN

$$
\boldsymbol{u}(t)=-(\boldsymbol{C B})^{-1}\left[\boldsymbol{C} \boldsymbol{A}_{i} \boldsymbol{x}(t)+\|N(t)\|\left\|\dot{h}_{\alpha}\right\|\|\boldsymbol{x}(t)\| \operatorname{sgn}(\boldsymbol{S})+\|\boldsymbol{C B}\|\left(\hat{\rho}_{0}+\hat{\rho}_{1}\|\boldsymbol{x}(t)\|\right) \operatorname{sgn}(\boldsymbol{S})+\varepsilon \operatorname{sgn}(\boldsymbol{S})\right]
$$

The global fuzzy VSC and the adaptation laws are designed as

$$
\boldsymbol{u}(t)=-\sum_{i=1}^{r} h_{i}(z)(\boldsymbol{C B})^{-1}\left[\boldsymbol{C} \boldsymbol{A}_{i} \boldsymbol{x}(t)+\hat{\varphi}(t, \boldsymbol{x}) \operatorname{sgn}(\boldsymbol{S})\right]
$$

with the following adaptive laws 


$$
\left\{\begin{array}{l}
\hat{\varphi}(t, \boldsymbol{x})=\|N(t)\|\left\|\dot{h}_{\alpha}\right\|\|\boldsymbol{x}(t)\|+\sum_{k=0}^{1} \hat{\rho}_{k}\|\boldsymbol{C} \boldsymbol{B}\|\|\boldsymbol{x}(t)\|^{k}+\varepsilon \\
\dot{\hat{\rho}}_{0}=\gamma_{0}\left\|\boldsymbol{S}^{T}(t)\right\|\left\|\sum_{i=1}^{r} h_{i}(z) \boldsymbol{C} \boldsymbol{B}_{i}\right\| \\
\dot{\hat{\rho}}_{1}=\gamma_{1}\left\|\boldsymbol{S}^{T}(t)\right\|\left\|\sum_{i=1}^{r} h_{i}(z) \boldsymbol{C} \boldsymbol{B}_{i}\right\|\|\boldsymbol{x}(t)\|
\end{array}\right.
$$

where the adaptation rates $\gamma_{i}>0$, the constant $\varepsilon>0$, and the functions $N(t), h_{\alpha}$ will be defined in the following theorem, which gives the stability analysis when the controller (30) is enforced on the system (4) and the reachability of the specified sliding surface $\boldsymbol{S}(t)=0$ can be obtained.

Theorem 2. For the uncertain fuzzy systems (4) with the switching function (5), $\boldsymbol{Q}$ is the feasible solution of LMIs (6). Then it can be shown that the state trajectories of the system (4) will be driven onto the switching surface $\boldsymbol{S}(t)=0$ and asymptotically converge to zero by the adaptive SMC law in (20),(21) and (22).

Proof: For purpose of design integrity, a simple stability analysis based on Lyapunov direct method is carried out. Define the Lyapunov function candidate

$$
V_{2}(t)=\frac{1}{2} \boldsymbol{S}^{T} \boldsymbol{S}+\sum_{k=0}^{1} \frac{1}{2 \gamma_{k}} \tilde{\rho}_{k}^{2} \geq 0
$$

where the estimation error $\tilde{\rho}_{k}=\hat{\rho}_{k}-\rho_{k}$.

By differentiating the switching surface $\boldsymbol{S}(t)$, we have

$$
\dot{\boldsymbol{S}}(t)=\boldsymbol{C}(t) \dot{\boldsymbol{x}}(t)+\dot{\boldsymbol{C}}(t) \boldsymbol{x}(t)
$$

In (24), by following from Lemma 1 we have

$$
\dot{\boldsymbol{C}}(t)=\frac{d}{d t}\left(h_{\alpha}^{T} \boldsymbol{P} h_{\alpha}\right)^{-1} \boldsymbol{B}_{\alpha} h_{\alpha}=N(t) \dot{h_{\alpha}}(t)
$$

where $h_{\alpha}(t)=\left[h_{1}, \ldots, h_{r}\right], \boldsymbol{B}_{\alpha}(t)=\left[\boldsymbol{B}_{1}, \ldots, \boldsymbol{B}_{r}\right]^{T}, \boldsymbol{P}=\boldsymbol{B}_{\alpha} \boldsymbol{Q}^{-1} \boldsymbol{B}_{\alpha}^{T}, N(t)=\left[-\left(h_{\alpha}^{T} \boldsymbol{P} h_{\alpha}\right)^{-1} h_{\alpha}^{T}(\boldsymbol{P}+\right.$ $\left.\left.\boldsymbol{P}^{T}\right)\left(h_{\alpha}^{T} \boldsymbol{P} h_{\alpha}\right)^{-1} \boldsymbol{B}_{\alpha} h_{\alpha}+\left(h_{\alpha}^{T} \boldsymbol{P} h_{\alpha}\right)^{-1} \boldsymbol{B}_{\alpha}\right]$

Then, it follows from (4) that we have

$$
\dot{\boldsymbol{S}}(t) \boldsymbol{S}(t)=\boldsymbol{C}(t) \dot{\boldsymbol{x}}(t) \boldsymbol{C}(t) \boldsymbol{x}(t)+N(t) \dot{h_{\alpha}}(t) \boldsymbol{x}(t) \boldsymbol{C}(t) \boldsymbol{x}(t)
$$

By substituting the controller (21) into (26), we have

$$
\begin{aligned}
\boldsymbol{S}^{T}(t) \dot{\boldsymbol{S}}(t)= & \boldsymbol{S}^{T}(t)\left[\sum_{i=1}^{r} h_{i}(z) \boldsymbol{C} \boldsymbol{A}_{i} \boldsymbol{x}(t)+\boldsymbol{C} \boldsymbol{B} \boldsymbol{u}(t)+N(t) \dot{h}_{\alpha}(t) \boldsymbol{x}(t)+\sum_{i=1}^{r} h_{i}(z) \boldsymbol{C} \boldsymbol{B}_{i} g(t, \boldsymbol{x}, \boldsymbol{u})\right] \\
& =\boldsymbol{S}^{T}(t)\left[N(t) \dot{h}_{\alpha}(t) \boldsymbol{x}(t)+\boldsymbol{C} \boldsymbol{B} g(t, \boldsymbol{x}, \boldsymbol{u})-\hat{\varphi}(t, \boldsymbol{x}) \operatorname{sgn}(\boldsymbol{S}(t))\right] \\
& \leq\left\|\boldsymbol{S}^{T}(t)\right\|\|N(t)\|\left\|\dot{h}_{\alpha}\right\|\|\boldsymbol{x}(t)\|+\left\|\boldsymbol{S}^{T}(t)\right\|\|\boldsymbol{C} \boldsymbol{B}\| \sum_{k=0}^{1} \rho_{k}\|\boldsymbol{x}(t)\|^{k}-\hat{\varphi}(t, \boldsymbol{x})\|\boldsymbol{S}(t)\| \\
& =\| \boldsymbol{S}^{T}(t)\left[\|\boldsymbol{C} \boldsymbol{B}\| \sum_{k=0}^{1} \rho_{k}\|\boldsymbol{x}(t)\|^{k}-\|\boldsymbol{C} \boldsymbol{B}\| \sum_{k=0}^{1} \hat{\rho}_{k}\|\boldsymbol{x}(t)\|^{k}-\varepsilon\right]
\end{aligned}
$$


By differentiating the function $V_{2}$ and substituting the adaptive laws (22) into (27), the simplified expression of (27) can be obtained as

$$
\dot{V}_{2}(t)=\boldsymbol{S}^{T} \dot{\boldsymbol{S}}+\frac{\dot{\tilde{\rho}}_{0}}{\gamma_{0}}\left(\hat{\rho}_{0}-\rho_{0}\right)+\frac{\dot{\tilde{\rho}}_{1}}{\gamma_{1}}\left(\hat{\rho}_{1}-\rho_{1}\right)=-\varepsilon\left\|\boldsymbol{S}^{T}(t)\right\|
$$

Noticing that $\epsilon>0$, thus the derivative $\dot{V}_{2}(t)<0$ when $\boldsymbol{S}(t) \neq 0$, which implies that under the controller (21) and (22) the reachability of the specified switching surface is guaranteed, and the trajectories of the fuzzy uncertain system (3) are globally driven onto the specified switching surface $\boldsymbol{S}(t) \neq 0$. Moreover, it is seen that the estimation error $\tilde{\rho}_{k}$ will converge to zero.

\section{Numerical Simulation}

To show the effectiveness of the proposed controller design techniques, the inverted pendulum with parametric uncertainties, which is taken from $\mathrm{Wu}$ and Juang [16], is formulated for simulation. The control objective is to drive its state trajectories to the origin. The equations of motion for the inverted pendulum device are

$$
\left\{\begin{array}{l}
\dot{x}_{1}(t)=x_{2}(t) \\
\dot{x}_{2}(t)=\frac{-f_{1} m l x_{2} \cos x_{1}-m^{2} g l^{2} \sin x_{1} \cos x_{1}+\left(J+m l^{2}\right)\left[m l x_{2}^{2} \sin x_{1}-f_{0} x_{4}+u(t)+d(t)\right]}{\bar{M}\left(J+m l^{2}\right)-m^{2} l^{2} \cos ^{2} x_{1}} \\
\dot{x}_{3}(t)=x_{4}(t) \\
\dot{x}_{4}(t)=\frac{-f_{1} \bar{M} x_{2}^{2}-m^{2} l^{2} x_{2}^{2} \sin x_{1} \cos x_{1}+f_{0} m l x_{4} \cos x_{1}+\bar{M} m g \sin x_{1}-m l \cos x_{1}[u(t)+d(t)]}{\bar{M}\left(J+m l^{2}\right)-m^{2} l^{2} \cos ^{2} x_{1}}
\end{array}\right.
$$

where $x_{1}$ denotes the angle ( $\mathrm{rad}$ ) of the pendulum from the vertical, $x_{2}$ is the angular velocity $(\mathrm{rad} / \mathrm{s}), x_{3}$ is the displacement $(\mathrm{m})$ of the cart, and $x_{4}$ is the velocity of the cart. $g=9.8 \mathrm{~m} / \mathrm{s}^{2}$ is the gravity constant, $m$ is the mass $(\mathrm{kg})$ of the pendulum, $M$ is the mass $(\mathrm{kg})$ of the cart, $f_{0}$ is the friction factor $(\mathrm{N} / \mathrm{m} / \mathrm{s})$ of the cart, $f_{1}$ is the friction factor $(\mathrm{N} / \mathrm{rad} / \mathrm{s})$ of the pendulum, $l$ is the length $(\mathrm{m})$ from the center of the mass of the pendulum to the shaft axis, $J$ is the moment of inertia (kg.m2) of the pendulum round its center of mass, and $u(t)$ is the force $(\mathrm{N})$ applied to the cart. The model parameters are given as: $\bar{M}=M+m, M=1.3282 \mathrm{~kg}, m=0.22 \mathrm{~kg}$, $f_{0}=22.915 \mathrm{~N} / \mathrm{m} / \mathrm{s}, f_{1}=0.007056 \mathrm{~N} / \mathrm{rad} / \mathrm{s}, l=0.304 \mathrm{~m}, J=0.004963 \mathrm{~kg} . \mathrm{m} 2$ in the numerical simulation. It's assumed that $d(t)$ is bounded by $d(t) \leq \rho_{0}+\rho_{1}\|\boldsymbol{x}\|$, where $\rho_{i}$ is unknown parameter. The fuzzy model of system is described as the following two rules:

Plant rule $i$ : IF $x_{1}(t)$ is $M_{1}^{i}$, THEN $\dot{\boldsymbol{x}}(t)=\boldsymbol{A}_{i}+\boldsymbol{B}_{i}[u(t)+g(t, \boldsymbol{x})$.

The model parameters are given as $\boldsymbol{A}_{1}=\left[\begin{array}{cccc}0 & 1 & 0 & 0 \\ 29.2529 & -0.3149 & 0 & 44.1811 \\ 0 & 0 & 0 & 1 \\ -1.2637 & 0.0136 & 0 & -16.7096\end{array}\right], \boldsymbol{A}_{2}=$ $\left[\begin{array}{cccc}0 & 1 & 0 & 0 \\ 22.0587 & -0.2872 & 0 & 20.1425 \\ 0 & 0 & 0 & 1 \\ -0.4765 & 0.0062 & 0 & -15.2361\end{array}\right], \boldsymbol{B}_{1}=\left[\begin{array}{c}0 \\ -1.9280 \\ 0 \\ 0.7292\end{array}\right], \boldsymbol{B}_{2}=\left[\begin{array}{c}0 \\ -0.8790 \\ 0 \\ 0.6649\end{array}\right]$. The membership functions are selected as $M_{1}(\boldsymbol{x})=\left[1-1 /\left(1+e^{-7\left(x_{1}-\pi / 24\right)}\right)\right] /\left(1+e^{-7\left(x_{1}+\pi / 24\right)}\right), M_{2}(\boldsymbol{x})=$ $1-M_{1}(\boldsymbol{x})$.Due to $\boldsymbol{B}_{2} \leq \boldsymbol{B}_{1}$, the stabilization result (Zheng et al., 2002) is invalid. The perturbation is set to be $g(t, \boldsymbol{x})=[0.1 \text { sint }, 0.05 \sin t]^{T}$, the initial states $\boldsymbol{x}(0)=[\pi / 3,0, \pi / 5,0]$. To assess 
the effectiveness of our fuzzy controller, we apply the controller to the original system (12) with nonzero $d(t)$. We choose the adaptation parameters $\gamma_{0}=0.001, \gamma_{1}=0.1$.Via LMI optimization with (19), we obtain the feasible solutions and the switching suafrace.

The simulation results are given in Figures 1-2. It is seen that the reachability of the sliding motion can be guaranteed. The system enters sliding-mode motion after about $t=0.8$ second. From Figure 1, one can see that the system states converge to zero fast, furthermore, the simulation results also show that our present design effectively attenuates the effect of both parameter uncertainties and external disturbances. In Figure 2, the control effort is shown and approaches to be stable after a short-term adjustment in the initial stage.

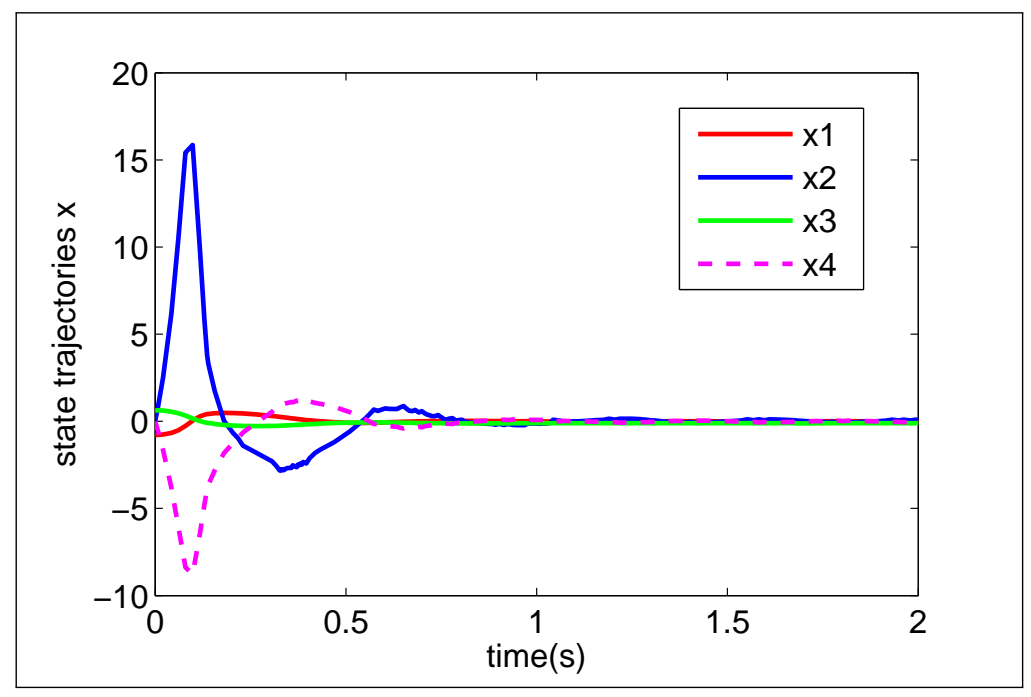

Figure 1: Trajectories of states $x_{1}, x_{2}, x_{3}$ and $x_{4}$

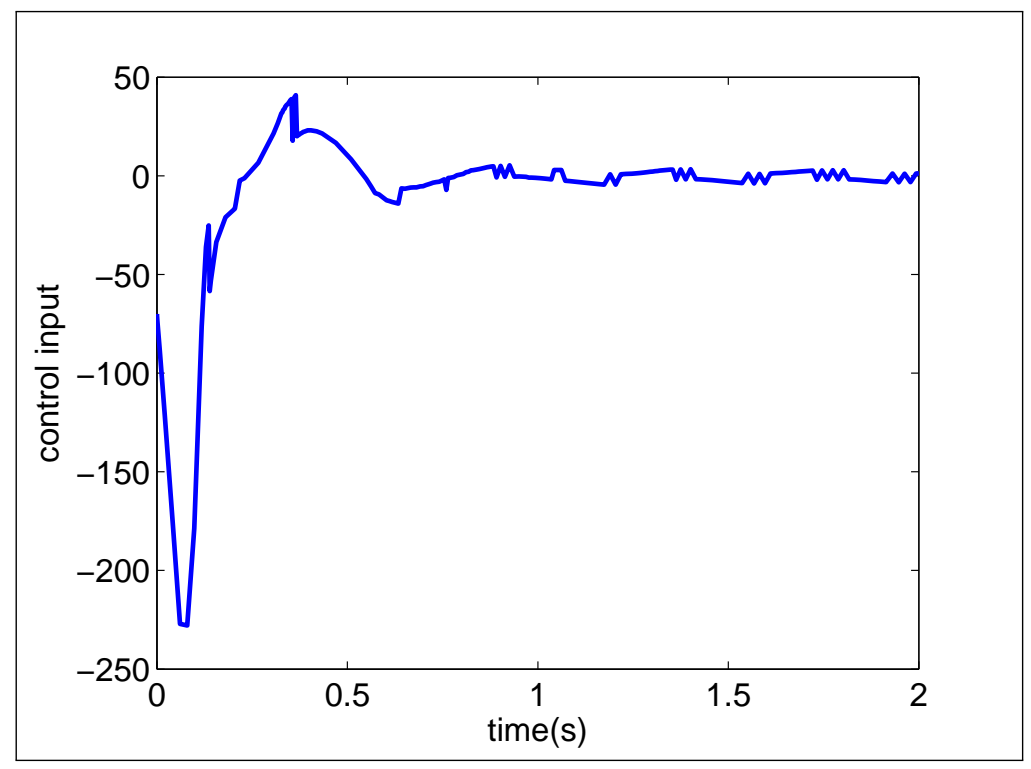

Figure 2: Control input 


\section{Conclusions and Future Works}

This paper has generalized the T-S fuzzy model to represent a class of nonlinear systems which includes parameter uncertainties or external disturbances. A novel adaptive VSC control scheme has been proposed for the uncertain model, which relaxes the restrictive assumption that the input matrices of the local sub-models are identical and needs no information of uncertainties. The overall fuzzy VSC controller of the system is achieved by fuzzy blending of the local VSC controller. The existence condition of linear sliding surfaces guaranteeing asymptotic stability of the equivalent dynamics is derived as well as the stability analysis. Finally, a numerical design example is illustrated in order to show the effectiveness of our scheme.

\section{Acknowledgment}

The authors are grateful to the support of the National Natural Science Foundation of China (61203019), the China Postdoctoral Science Foundation funded project (2012M521518), the Key Projects of Chinese Ministry of Education (No.212122), the Department of Education (14A032) and Innovative Research Team in Higher Educational Institutions of Hunan Province, and the Natural Science Foundation of Hunan Provincial (No.13JJ9019).

\section{Bibliography}

[1] Takagi, T.; Sugeno, M. (1985); Fuzzy identification of systems and its applications to modeling and control, IEEE Trans. Syst., Man, Cybern., ISSN 0018-9472, 15(1): 116-132.

[2] Feng, G. (2006); A survey on analysis and design of model-based fuzzy control systems, IEEE Trans. Fuzzy Syst., ISSN 1063-6706, 14(5): 676-697.

[3] Tanaka, K.; Ikeda, T.; Wang, H.O. (1998); Fuzzy regulators and fuzzy observers: Relaxed stability conditions and LMI-based designs, IEEE Trans. Fuzzy Syst., ISSN 1063-6706, 6(2): 250-265.

[4] Zhang, X. Z.; Wang, Y. N. (2009); Fuzzy variable structure control based on a TakagiSugeno model for permanent magnet synchronous motors, Journal of Systems and Control Engineering, ISSN 0959-6518, 223(6): 773-783.

[5] Besheera, A. H.; Emarab, H. M.; Azizb, M. M. A.(2009); Wind energy conversion system regulation via LMI fuzzy pole cluster approach, Electric Power System Research, ISSN 03787796, 79(6): 531-538.

[6] Aouaouda, S.; Chadli, M.et.al.(2012); Robust fault tolerant tracking controller design for unknown inputs TS models with un-measurable premise variables, Journal of Process Control, ISSN 0959-1524, 22(5): 861-872.

[7] Liang, Y. W.; Xu, S. D.; Liaw, D. C.et.al.(2008); A study of T-S model-based SMC scheme with application to robot control, IEEE Trans. Ind.Electron., ISSN 0278-0046, 55(11): 39643971.

[8] Tian, E. G.; Peng, C.(2006); Delay dependent stability analysis and synthesis of uncertain TS fuzzy systems with time-varying delay, Fuzzy Sets and Systems, ISSN 0165-0114, 157(4): 544-559. 
[9] Wu, H. N.(2007); Robust H2 fuzzy output feedback control for discrete-time nonlinear systems with parametric uncertainties, International Journal of Approximate Reasoning, ISSN 0888613X, 46(1): 151-165.

[10] Lin, Y.C.; Lo, J.C.(2005); Robust mixed H2/H $\infty$ filtering for discrete-time delay fuzzy systems, International Journal of System Science, ISSN 1841-9836, 36(15): 993-1006.

[11] Wang Y.N.; Zhang X.Z.et.al.(2011); Position-sensorless hybrid sliding mode control of electric vehicles with brushless DC motor, IEEE Trans. Veh. Technol., ISSN 0018-9545, 60(2): 421-432.

[12] Zheng, F.; Wang, Q. G. et.al.(2002); Output tracking control of MIMO fuzzy nonlinear systems using variable structure control approach, IEEE Trans. Fuzzy Syst., ISSN 1063-6706, 10(3): $686-697$.

[13] Cao, W. J.; Xu, J. X.(2004); Nonlinear integral-type sliding surface for both matched and unmatched uncertain systems, IEEE Trans. Automat. Contr., ISSN 0018-9286, 49(8): 13551360 .

[14] Choi, H. H.(2008); Robust stabilization of uncertain fuzzy systems using variable structure system approach, IEEE Trans. Fuzzy Syst., ISSN 1063-6706, 16(3): 715-724.

[15] Zhang, X.Z.; Wang, Y.N.et.al.(2010); H $\infty$ robust T-S fuzzy design for uncertain nonlinear systems with state delays based on sliding-mode control, International Journal of Computers Communications \& Control, ISSN 1841-9836, 5(4): 592-602.

[16] Wu, T.Z.; Juang, Y.T.(2008); Design of variable structure control for fuzzy nonlinear systems, Expert Systems with Applications, ISSN 0957-4174, 35(3): 1496-1503. 